\title{
Challenges for FCC-ee luminosity monitor design
}

\author{
Mogens Dam ${ }^{\mathrm{a}}$ \\ Niels Bohr Institute, Copenhagen University, Copenhagen, Denmark
}

Received: 5 May 2021 / Accepted: 4 December 2021

(C) The Author(s) 2021, corrected publication 2022

\begin{abstract}
For cross section measurements, an accurate knowledge of the integrated luminosity is required. The FCC-ee physics programme at and around the $\mathrm{Z}$ pole sets the ambitious precision goal of $10^{-4}$ on the absolute luminosity measurement and one order of magnitude better on the relative measurement between energy scan points. The luminosity is determined from the rate of Bhabha scattering, $\mathrm{e}^{+} \mathrm{e}^{-} \rightarrow \mathrm{e}^{+} \mathrm{e}^{-}$, where the final state electrons and positrons are detected in dedicated monitors covering small angles from the outgoing beam directions. The constraints on the luminosity monitors are multiple: (i) they are placed inside the main detector volume only about $1 \mathrm{~m}$ from the interaction point; (ii) they are centred around the outgoing beam directions and do not satisfy the normal axial detector symmetry; (iii) their coverage is limited by the beam pipe, on the one hand, and by the requirement to stay clear of the main detector acceptance, on the other; (iv) the steep angular dependence of the Bhabha scattering process imposes a precision on the acceptance limits at about $1 \mu \mathrm{rad}$, corresponding to an absolute geometrical precision of $\mathcal{O}(1 \mu \mathrm{m})$ on the monitor radial dimensions; and $v$ ) the very high bunch-crossing rate of $50 \mathrm{MHz}$ during the Z-pole operation calls for fast readout electronics. Inspired by second-generation LEP luminosity monitors, which achieved an experimental precision of $3.4 \times 10^{-4}$ on the absolute luminosity measurement (Abbiendi et al. in Eur Phys J C 14:373-425, 2000), a proposed ultra-compact solution is based on a sandwich of tungsten-silicon layers. A vigorous $\mathrm{R} \& \mathrm{D}$ programme is needed in order to ensure that such a solution satisfies the more challenging FCC-ee requirements.
\end{abstract}

\section{Introduction}

The integrated luminosity acts as the link between the number of events $N$ arising from a given physics process and the corresponding cross section $\sigma$ :

$$
N=L \cdot \sigma .
$$

Precise cross section measurements therefore depend on the accurate knowledge of the luminosity. At the Large Hadron Collider (LHC), the main method for luminosity measurement is via direct calculations based on known beam parameters, with important input on transverse beam profiles from so-called van der Meer scans. Precisions at the few percent level are reached using this method [1]. In electron-positron collisions, the luminosity is usually inferred by exploiting Eq. (1) for a reference process with a precisely known cross section,

\footnotetext{
a e-mail: dam@nbi.dk (corresponding author)
} 
$\sigma_{\text {ref }}$. This way, cross section measurements are, in fact, based on the ratio of event counts between the physics process under study and the reference process, $\sigma=\left(N / N_{\text {ref }}\right) \cdot \sigma_{\text {ref }}$. In order for measurements not to be limited by the statistical uncertainty on $N_{\text {ref }}$, the reference process should be chosen with a sufficiently large cross section, $\sigma_{\text {ref }} \gtrsim \sigma$. At the recent generation of $\mathrm{e}^{+} \mathrm{e}^{-}$colliders, the B-factories operating at centre-of-mass energies around the $\Upsilon(4 \mathrm{~S})$ resonance $(\sim 10 \mathrm{GeV})$, a sub-per-cent-level precision has been reached based on large-angle production of $\mathrm{e}^{+} \mathrm{e}^{-}, \gamma \gamma$, or $\mu^{+} \mu^{-}$final states [2,3].

Luminosity measurements with precisions at the sub-per-mil level were pioneered at LEP using small-angle Bhabha scattering, $\mathrm{e}^{+} \mathrm{e}^{-} \rightarrow \mathrm{e}^{+} \mathrm{e}^{-}$. Events were detected by dedicated monitors encircling the beam at about $2.5 \mathrm{~m}$ from the interaction point. The small-angle Bhabha cross section can be calculated with high precision from quantum electrodynamics and depends only weakly on the properties of the $\mathrm{Z}$ boson, even at centre-of-mass energies close to the $\mathrm{Z}$ pole. To lowest order, the strongly forward-peaked cross section [4] takes the form

$$
\sigma=\frac{16 \pi \alpha^{2}}{s}\left(\frac{1}{\theta_{\min }^{2}}-\frac{1}{\theta_{\max }^{2}}\right),
$$

for a detector with a polar angle coverage from $\theta_{\min }$ to $\theta_{\max }$ and full coverage in azimuth. During the first period of LEP operation, it was realised that the number of $\mathrm{Z} \rightarrow$ hadrons decays could be measured with systematic uncertainty well below the per-mil level. An upgrade of the luminosity monitors, originally designed for per-cent-level measurements, therefore became desirable. Upgrades took different form in the four experiments: in L3, a precise Si tracker was added in front of their BGO calorimeter [5]; in DELPHI, the original SPACAL-like calorimeter was replaced by a more precise device based on scintillating tiles [6]; and in OPAL and ALEPH, new compact SiW-sandwich calorimeters were added in front of the original devices covering the lowest scattering angles $[7,8]$.

To appreciate the extraordinary efforts that were invested in reaching sub-per-mil levels of accuracy, it is worthwhile consulting the work by OPAL, described in detail in Ref. [7], resulting in an experimental precision as low as $3.4 \times 10^{-4}$, more than twice better than the LEP competitors. At this stage, dominant contributions to the remaining uncertainty included: (i) radial metrology of the calorimeters $\left(1.4 \times 10^{-4}\right)$, (ii) uncertainty on the correspondence between measured shower coordinates and true scattering angles $\left(1.4 \times 10^{-4}\right)$, (iii) calorimeter energy response $\left(1.8 \times 10^{-4}\right)$, and (iv) clustering algorithm $\left(1.0 \times 10^{-4}\right)$. Smaller contributions were associated with $(v)$ beam-related backgrounds $\left(0.8 \times 10^{-4}\right)$ and (vi) beam parameters $\left(0.6 \times 10^{-4}\right)$.

At FCC-ee, precise luminosity measurement will again be of vital importance. The FCC-ee programme [9] includes four major phases with precision measurements of the four heaviest particles of the Standard Model: $(i)$ the $\mathrm{Z}$ boson, with $5 \times 10^{12} \mathrm{Z}$ decays collected around the $\mathrm{Z}$ pole, (ii) the $\mathrm{W}$ boson, with $10^{8} \mathrm{WW}$ pairs collected close to threshold, (iii) the Higgs boson, with $1.2 \times 10^{6} \mathrm{e}^{+} \mathrm{e}^{-} \rightarrow \mathrm{HZ}$ events produced at the cross section maximum, and (iv) the top quark, with $10^{6}$ tt pairs produced at and slightly above threshold. In particular, the first two phases, with their superior statistics, call for the highest achievable systematic accuracy $[10,11]$. Ambitious precision goals have been set to $10^{-4}$ for the absolute luminosity measurement and one order of magnitude better for the relative measurement between energy scan points. These goals match well the forecast for the achievable theoretical precision of $10^{-4}$ on the small-angle Bhabha process [12]. As at LEP, the luminosity measurement is foreseen to be principally based on small-angle Bhabha scattering. Bhabha scattering may be complemented by large-angle $\mathrm{e}^{+} \mathrm{e}^{-} \rightarrow \gamma \gamma$ production, for which, despite a three-orders- 
of-magnitude smaller rate, the statistical precision is sufficient for most purposes, and the systematic uncertainties are expected to be largely independent.

This essay concentrates on the luminosity measurement based on small-angle Bhabha scattering. Building on the experience from the LEP experiments, it discusses a possible design of the luminosity monitors and the measurement methodology in order to illustrate the challenges presented by the precise FCC-ee luminosity goals. Emphasis is placed on deriving the required geometrical precisions of the monitors and of their alignment with respect to the accelerator. The design described is that developed for the FCC-ee Conceptual Design Report [13].

\section{Experimental environment}

The experimental environment at FCC-ee is described in detail in the CDR [13]. The FCC-ee accelerator has been designed to provide optimal luminosities at all energies from the $\mathrm{Z}$ pole to the $\mathrm{t} \overline{\mathrm{t}}$ production threshold and above. Of direct relevance to the luminosity measurement, the design includes: $(i)$ separate storage rings for the electron and positron beams, which are brought into collision at a $30 \mathrm{mrad}$ horizontal crossing angle at two (possibly four) interaction points (IPs); (ii) strong vertical focusing of the beams provided by a set of quadrupoles, all the way to $L^{*}=2.2 \mathrm{~m}$ from the IP; and (iii) very high beam crossing rates of $\mathcal{O}(50 \mathrm{MHz})$ for the operation at and around the $\mathrm{Z}$ pole. A detector is placed at each IP, with a solenoid that delivers a magnetic field of $2 \mathrm{~T}$, parallel to the bisector of the two beams, the $z$-axis. Two complementary detector concepts have been studied. In both cases, the trajectories of charged particles are measured within a tracker down to polar angles of about $150 \mathrm{mrad}$ with respect to the $z$-axis. The tracker is surrounded by a calorimeter and muon-detection system. The region covering polar angles below $100 \mathrm{mrad}$ corresponds to the "machine-detector interface" (MDI), the design of which demands special care. The crossing of the beams at an angle of $\pm 15 \mathrm{mrad}$ with the detector field necessitates the insertion of a set of one-meter-long compensating solenoidal magnets in front of the quadrupoles in order to preserve the low vertical beam emittance. This arrangement pushes the luminosity monitors far into the main detector volume, where the available space is severely constrained. A brief account of the MDI can be found in Ref. [14], from which Fig. 1, showing a sketch of the interaction region, is extracted.

\section{Luminosity monitor design}

A set of SiW calorimeters is proposed as luminosity monitors at FCC-ee. In addition to providing a very compact solution, the choice of a purely calorimetric measurement also has theoretical advantages. As it has been pointed out by the authors of the higher-order Bhabha event generator BHLUMI [15], there is theoretical difficulty in ascribing meaning to the trajectory of a bare Bhabha-scattered electron. Theoretically more meaningful is the concept of a dressed electron, i.e. an electron including its close-lying radiated photons, thus favouring a calorimetric measurement.

Following the examples from OPAL [7] and ALEPH [8] and from linear collider studies $[16,17]$, the proposed calorimeters have been designed as cylindrical devices assembled from stacks of identical SiW layers. This simple geometry facilitates the control of construction and metrology tolerances to the necessary micron level. To measure precisely the scattering angles of the Bhabha-scattered electrons and positrons, the calorimeters are centred around-and 


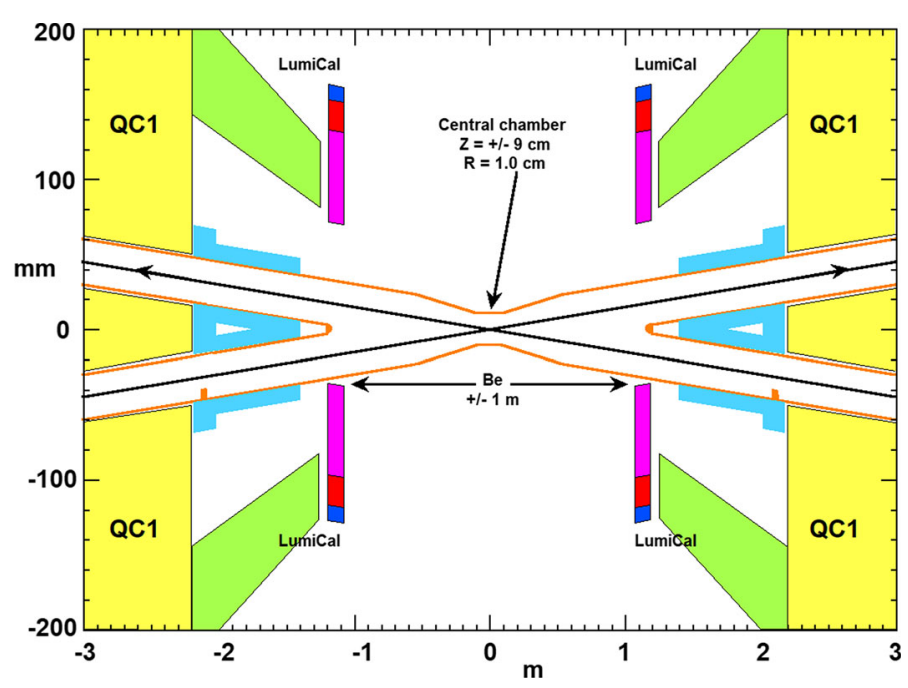

Fig. 1 From Ref. [14]: Sketch of the interaction region in the horizontal plane. Compensating solenoids (green) are installed in front of the quadrupoles (yellow) to compensate for the effect of the detector field during the traversal of the beams at a $15 \mathrm{mrad}$ angle. The luminosity calorimeters (LumiCal) are installed in front of the compensating solenoids and centred around the outgoing beam directions

tilted to be perpendicular to- the outgoing beam directions. The physical dimensions of the calorimeters, installed in front of the compensating solenoids extending to $|z| \simeq 1.2 \mathrm{~m}$ from the IP, are limited by two parameters: At their inner radius, they must stay clear of the incoming beam pipe; at their outer radius, they must stay away from the tracker acceptance defined by a 150-mrad cone around the $z$-axis. The situation is illustrated in Fig. 2, where also the main physical dimensions of the proposed design are given. The design includes 25 layers, each layer comprising a 3.5-mm tungsten plate, equivalent to $1 X_{0}$, and a Si-sensor plane inserted in the 1-mm gap. In the transverse plane, the Si sensors are finely partitioned into pads. The proposed number of divisions is 32 both radially and azimuthally for 1024 readout channels per layer, or 25600 channels in total for each calorimeter. A 30-mm uninstrumented region at the outer circumference is reserved for services. This region includes front-end electronics, cables, and cooling, and, importantly, also the physical structures-likely including precision dowels and bolts - for the assembly of the SiW sandwich. It should be emphasised that a proper engineering design has so far not been done but is highly needed in order to verify the proposed assembly procedure and the corresponding necessary space at the outer radius. Overall, the proposed design is very compact, each calorimeter weighing only about $65 \mathrm{~kg}$.

In the base design, each calorimeter is divided vertically into two half-barrels clamped together around the beam pipe. Due to the small dimensions, it may be possible to produce each silicon half-layer from a single silicon tile, which will minimise potential inactive regions between sensors and facilitate the precise geometrical control of the acceptance. Meticulous care is required for the design of the vertical assembly of the two half-barrels, both in order to avoid a non-instrumented region and to precisely control the geometry. An alternate design has been proposed, where the monitors would be built as full barrels avoiding the vertical assembly. This solution would have strong implications for the design of the MDI and the assembly procedure of the detector around the beam pipe. 

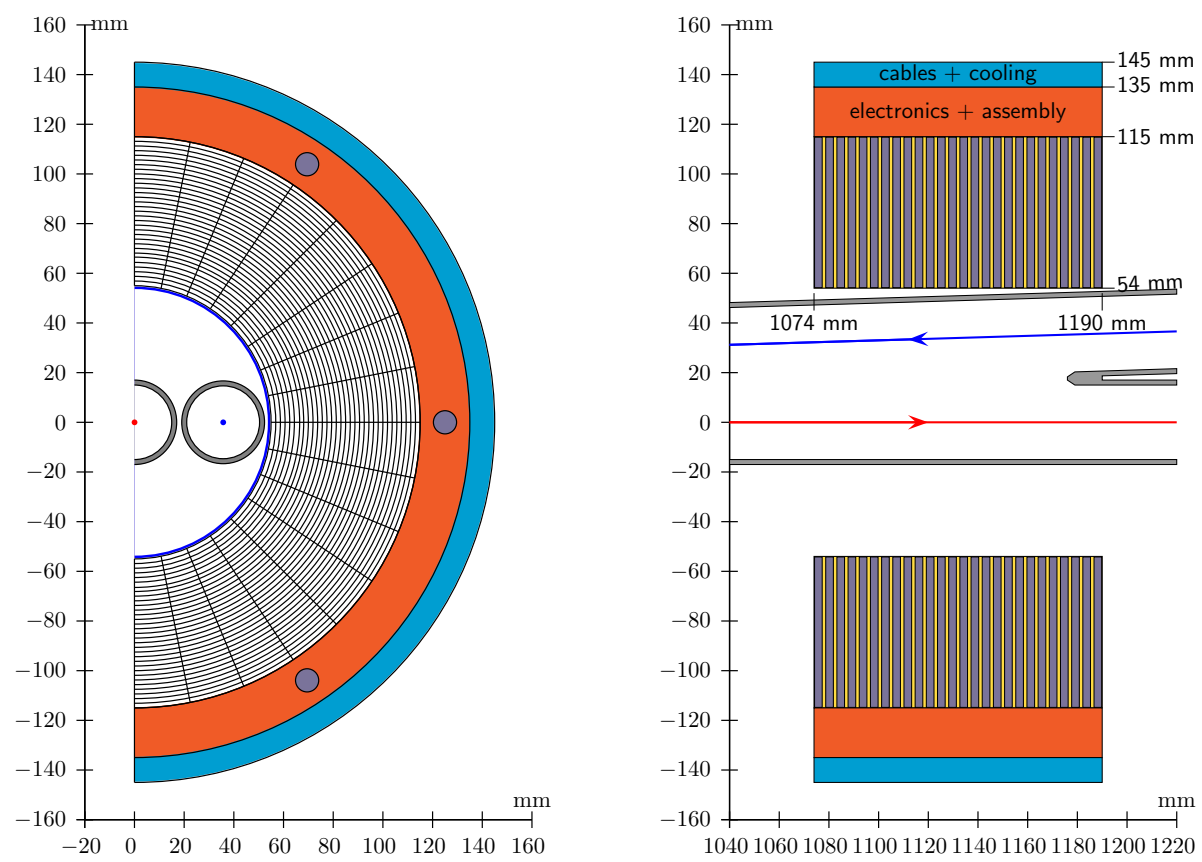

Fig. 2 From Ref. [13]: A possible layout for the luminosity calorimeter centred around the outgoing beam direction (shown as a red arrow): front view (left), top view (right). The sensitive region, instrumented with silicon sensors, is displayed in black on the front view, the region for front-end electronics in red, and the region for cables and cooling in blue. The top view shows the interleaved silicon (yellow)-tungsten (purple) layer structure of the sensitive region

The Si-sensor pads will be connected to compact front-end electronics positioned at radii immediately outside the sensors. To minimise the occurrence of pile-up events-at the $\mathrm{Z}$ pole, the average number of Bhabha events hitting the luminometers per bunch crossing is about $10^{-3}$ - it is desirable to read out the detector at the bunch-crossing rate. This requirement calls for the development of readout electronics with a shaping time below the bunch spacing of $20 \mathrm{~ns}$. A power budget of $5 \mathrm{~mW}$ per readout channel has been estimated, for a total of $130 \mathrm{~W}$ per calorimeter, to be removed via cooling. With thermal expansion coefficients of both tungsten and Si-crystal at the few $\times 10^{-6} /{ }^{\circ} \mathrm{C}$ level, control of the monitors' temperature within a $1{ }^{\circ} \mathrm{C}$ tolerance would seem appropriate for control of physical dimensions to the 1- $\mu \mathrm{m}$ level. A somewhat higher thermal sensitivity was observed in OPAL [7] and was caused by the aluminium structures supporting the tungsten absorbers. As in OPAL, temperatures should be continuously monitored and gradients should be minimised.

The large number of Bhabha events expected during FCC-ee operation around the $\mathrm{Z}$ pole will contribute an integrated radiation dose of about $0.01 \mathrm{MGy}(1 \mathrm{MRad})$, for the innermost ring of Si pads near shower maximum. This dose is supplemented by the contribution from beamstrahlung, where according to studies based on the GuineaPig simulation code [18], each calorimeter will see about $0.1 \mathrm{GeV}(3 \mathrm{GeV})$ of energy from beamstrahlung per bunch crossing at $\sqrt{s}=91.2$ (365) GeV. In terms of radiation dose, the higher beamstrahlung activity at higher collision energies is compensated by the lower bunch-crossing frequency. Integrated over the FCC-ee running phases, the radiation dose from beamstrahlung is esti- 
mated to exceed that from Bhabha scattering by less than an order of magnitude resulting in a total dose very significantly below the $1.5 \mathrm{MGy}$ expected for the Si sensors of the CMS High Granularity Calorimeter [19]. Radiation doses are expected to fall off considerably at larger radii, where readout electronics, possibly including optical links, will be located. For the technology choices of sensors and front-end electronics, radiation issues will have to be revisited in light of the final design and parameters of the accelerator.

\section{Acceptance and geometrical precisions}

With the luminosity monitors centred around the outgoing beam directions, the monitors measure directly the scattering angles of the Bhabha-scattered electrons and positrons (with respect to their unscattered directions). Henceforth, the $30 \mathrm{mrad}$ crossing angle of the colliding beams plays no significant rôle for the measurement methodology as it is outlined below.

The proposed luminometer design has a full-depth coverage for scattering angles between 50 and $97 \mathrm{mrad}$. For a robust energy measurement, the acceptance limits must be kept some distance away from these borders. Choosing this distance to be $15 \mathrm{~mm}$, corresponding to one Molière radius, limits the acceptance to the $62-88 \mathrm{mrad}$ range. To ensure that the luminosity measurement has no first-order dependence on possible misalignments and movements of the beam system relative to the luminometer system (both in position and angle), the method of asymmetric acceptance, first introduced in Ref. [20] and later extensively used at LEP, will be employed. Bhabha events are selected if the $\mathrm{e}^{ \pm}$is inside a narrow acceptance in one calorimeter, and the $\mathrm{e}^{\mp}$ is inside a wide acceptance in the other. A $2 \mathrm{mrad}$ difference between the wide and narrow acceptances is judged adequate to accommodate possible misalignments and movements of the IP. The narrow acceptance thus covers the 64-86 mrad angular range, corresponding to a Bhabha cross section of $14 \mathrm{nb}$ at the $\mathrm{Z}$ pole (compared to $40 \mathrm{nb}$ for $\mathrm{Z}$ production), equivalent to $6.4 \times 10^{-4}$ events per bunch crossing. Hence, contrary to the situation at LEP, where the Bhabha cross section was kept higher than that of $\mathrm{Z}$ production, here the opposite situation is imposed by the severely constrained geometrical conditions. With the enormous Z-pole statistics, this is not regarded as a serious limitation, and already at the off-peak energy points in the line-shape scan the Bhabha rate will be comparable to that of $\mathrm{Z}$ production.

The forward-peaked $1 / \theta^{3}$ angular distribution of the Bhabha process causes the luminosity measurement to be particularly sensitive to the determination of the angular coverage. The sensitivity can be determined by simple analytic calculations, using only the $1 / \theta^{3}$ dependence and the physical dimensions of the monitor system. The Bhabha acceptance $A$ is affected by any change $\Delta R_{\text {in }}\left(\Delta R_{\text {out }}\right)$ of the inner (outer) edge radial coordinate as follows:

$$
\frac{\Delta A}{A} \approx-\frac{\Delta R_{\text {in }}}{1.6 \mu \mathrm{m}} \times 10^{-4}, \quad \text { and } \quad \frac{\Delta A}{A} \approx+\frac{\Delta R_{\text {out }}}{3.8 \mu \mathrm{m}} \times 10^{-4} .
$$

Similarly, $A$ is affected by any change $\Delta Z$ of the half-distance between the effective planes defining the radial measurements in the two calorimeters:

$$
\frac{\Delta A}{A} \approx+\frac{\Delta Z}{55 \mu \mathrm{m}} \times 10^{-4} .
$$

For the latter effect, the $30 \mathrm{mrad}$ beam crossing angle makes the situation slightly more complicated. Now, with the two calorimeters centred on different axes, $Z$ shall be interpreted as $Z=\frac{1}{2}\left(Z_{1}+Z_{2}\right)$, where $Z_{1}$ and $Z_{2}$ are the distances, measured along the two outgoing beam axes, from the (nominal) IP to the two luminometers. Compared to LEP, the sensitivity 
to the radial and axial dimensions of the luminometer system is a factor $2-3$ higher. The main reason for this is the shorter distance between the IP and the monitors.

With the method of asymmetric acceptance, only second-order dependencies of $A$ remain on radial and longitudinal offsets $\delta r$ and $\delta z$ of the average IP position relative to the luminosity system. Again the strengths of these dependencies can be estimated analytically. However, longitudinal offsets imply an implicit cut into the acollinearity distribution of Bhabha events and thus a dependence on the amount of initial state radiation. To quantify this effect, a high-statistics study was performed based on the Bhabha event generator BHLUMI [15] and a parameterised detector response, where the energy of close-lying electrons and photons was collected into clusters. The study confirmed the second-order dependencies as long as offsets are sufficiently small to be covered by the difference between the wide and narrow acceptance definitions: in this case, offsets of up to $\delta r \approx 0.5 \mathrm{~mm}$ transversely and $\delta z \approx$ $20 \mathrm{~mm}$ longitudinally. Inside this range, the observed changes of the acceptance could be parameterised as

$$
\frac{\Delta A}{A} \approx+\left(\frac{\delta r}{0.6 \mathrm{~mm}}\right)^{2} \times 10^{-4} \text { and } \frac{\Delta A}{A} \approx-\left(\frac{\delta z}{6 \mathrm{~mm}}\right)^{2} \times 10^{-4} .
$$

Whereas the result for transverse offsets agrees well with the analytic estimate, it is interesting to notice that, for longitudinal offsets, the simulation result is larger (by a factor four) than that of the analytic estimate and even has the opposite sign, demonstrating the importance of radiative effects. The effect of a possible tilt of the luminometer system with respect to the beam reference system has a similar effect on $A$ as a transverse offset: in Eq. 5, one simply has to replace $\delta r$ with $Z \delta \alpha$, where $\alpha$ is the tilt angle. It should be stressed that such offsets and tilts give rise to asymmetries in the Bhabha counting rate either azimuthally (radial offsets and tilts) or between the two calorimeters (longitudinal offsets) and can therefore be monitored and corrected for directly from the data. No such possibility of correction from the data exists for the detector construction tolerances, $\Delta R$ and $\Delta Z$, discussed in the previous paragraph, which therefore need to be controlled via precise metrology and alignment. As a reference, OPAL [7] achieved control of the inner radius of their calorimeters to a precision of $4.4 \mu \mathrm{m}$ through precise metrology. Relative to OPAL, several uncertainty contributions are expected to vanish if each sensor layer can be made from a single silicon crystal. A dominant remaining contribution then stems from the knowledge and stability of the half-barrel separation, which was $1.9 \mu \mathrm{m}$ in the case of OPAL.

In summary, to reach a precision of $10^{-4}$ on the absolute luminosity measurement, the radial dimensions of the luminosity monitors have to be controlled to a precision of $\mathcal{O}(1 \mu \mathrm{m})$ (4.4 $\mu \mathrm{m}$ achieved by OPAL), whereas the half-distance between the two monitors has to be controlled to $\mathcal{O}(50 \mu \mathrm{m})(50-70 \mu \mathrm{m}$ achieved by OPAL). The requirements on the alignment of the luminometer system with respect to the average IP position are considerably more relaxed: accuracies of order $0.5 \mathrm{~mm}$ and $5 \mathrm{~mm}$ are called for in the radial and longitudinal directions, respectively.

\section{Other sources of systematics}

A complete survey and subsequent study of systematic effects are still to be pursued. Important contributions are expected from the sources identified by OPAL and already mentioned in the introduction: the calorimeter energy response, the clustering algorithm, and the correspondence between measured shower coordinates and true scattering angles. So far, only beam backgrounds and beam-beam effects have been examined for their influence on the 
luminosity measurement. Evidently, these studies shall be followed up when a better understanding of the accelerator behaviour becomes available.

At LEP, the primary source of background for the luminosity measurement was from coincidences of off-momentum particles generated by beam particles scattering with the residual gas in the beam pipe and deflected by the quadrupoles into the luminometers [7]. With its much stronger focusing, the ratio between the instantaneous luminosity and the beam current is much higher at FCC-ee than at LEP. It is therefore not surprising, as pointed out already in Ref. [13], that this background source appears negligible in FCC-ee. In the same reference, other sources of backgrounds such as those from incoherent pair production and from synchrotron radiation were likewise argued to be unimportant.

Electromagnetic effects caused by the very large charge densities of the FCC-ee beam bunches affect the colliding particles in several related ways. The final state electrons and positrons from small-angle Bhabha scattering are focused by the electromagnetic field of the opposing bunches leading to a sizeable bias of the luminometer acceptance that must be corrected for. This effect was studied in Ref. [21], where it was demonstrated that several sets of measurements can be used to control this bias, and that it therefore should not compromise the targeted precision. Interestingly, as recently pointed out in Ref. [22], this effect was already present at LEP, where it led to an underestimation of the luminosity measurement by about $0.1 \%$, significantly larger than the originally reported experimental uncertainties.

\section{Relative luminosity}

For the relative normalisation between energy scan points, geometrical effects, the main focus of this essay, tend to cancel (if their variation with time is sufficiently slow). In OPAL, dominant effects on the relative normalisation were related to the accelerator with contributions from beam-related backgrounds $\left(0.8 \times 10^{-4}\right)$ and beam parameters $\left(0.6 \times 10^{-4}\right)$. Even if it is believed that FCC-ee compared to LEP will have less beam-induced background and that the beam parameters (tilts and divergences) will have less freedom to vary over time (as an illustration the beam-pipe radius was $\sim 50 \mathrm{~mm}$ at LEP versus $15 \mathrm{~mm}$, or possibly $10 \mathrm{~mm}$, at FCC-ee), it will certainly be a formidable challenge to improve on LEP by one order of magnitude. Here, as is often the case, several systematic effects may in fact be of a statistical nature and may be reducible with the huge FCC-ee statistics.

\section{Outlook}

Much work is needed towards a consolidated luminometer design fulfilling the many severe requirements. For the cylindrical CDR design, discussed in this essay, important points include:

1. Engineering-level study of the proposed detector assembly method involving precision dowels and through-going bolts. This study shall take into account the required $\mathcal{O}(1 \mu \mathrm{m})$ geometrical precision on the radial coordinate.

2. Realistic estimate of the space needed for services at radii outside the sensitive region. This region shall be kept as transparent as possible by the use of light materials to minimise particle interactions.

3. Design of a procedure for maintaining and monitoring the geometrical accuracy of the monitors via precise metrology and alignment. 
4. Reevaluation of the expected radiation dose when the collider design and parameters are finalised and assessment of whether existing sensor technologies are adequate or whether further technological R\&D will be required.

5. Design of compact, low-power readout electronics preferentially allowing readout at the $50 \mathrm{MHz}$ bunch-crossing rate, including the transmission of signals away from the detectors.

6. Design of a cooling system allowing control of a constant and uniform temperature over the monitors. The required tolerances have to be developed.

7. Integration of the monitors into the MDI design including support structures, which isolate the monitors from possible movements and vibration of the accelerator magnet system.

More fundamental issues are related to the detector coverage, which is severely limited by the geometrical constraints arising from the placement inside the main detector volume in the very crowded forward region.

8. There is no coverage for scattering angles below $50 \mathrm{mrad}$, which goes against the general wish to close as hermetically as possible FCC-ee detectors down towards the beam axes. Certainly there is physical room at the inside of the CDR design to place instrumentation towards smaller scattering angles in all azimuthal directions except that of the incoming beam pipe. The question is how such irregular shaped instrumentation would be compatible with the $1 \mu \mathrm{m}$ level accuracy needed on the radial limits of the acceptance definition. Engineering studies are needed to address this question.

9. There is full-depth coverage only up to scattering angles of $96 \mathrm{mrad}$. In the main detector system, this corresponds to a polar angle of $81 \mathrm{mrad}$ in the azimuthal direction of the incoming beam, which is considerably lower than the agreed-upon boundary at $100 \mathrm{mrad}$ between the MDI and the detector. Hence, there is a risk that the forward electromagnetic calorimeter, with its face at $|z| \simeq 2.3 \mathrm{~m}$, will not be able to reach down to such small angles and provide the necessary overlap between the two systems. At the same time, the outermost "corner" of the CDR design, with the $30 \mathrm{~mm}$ reserved for services, reaches out to a polar angle of $150 \mathrm{mrad}$. Should it be necessary to extend the service region further, this risks to clash with the tracking system. There is a clear tension here, and the final design will have to be done taking the overall detector layout into account.

Acknowledgements I would like to thank Patrick Janot for proofreading and for helping to bring this manuscript into its final form.

Funding This project is co-funded from the European Union's Horizon 2020 research and innovation programme under grant agreement No 95175 .

Open Access This article is licensed under a Creative Commons Attribution 4.0 International License, which permits use, sharing, adaptation, distribution and reproduction in any medium or format, as long as you give appropriate credit to the original author(s) and the source, provide a link to the Creative Commons licence, and indicate if changes were made. The images or other third party material in this article are included in the article's Creative Commons licence, unless indicated otherwise in a credit line to the material. If material is not included in the article's Creative Commons licence and your intended use is not permitted by statutory regulation or exceeds the permitted use, you will need to obtain permission directly from the copyright holder. To view a copy of this licence, visit http://creativecommons.org/licenses/by/4.0/. 


\section{References}

1. P. Grafström, W. Kozanecki , Luminosity determination at proton colliders, Progress Particle Nuclear Phys. 81, 97-148 (2015). https://www.sciencedirect.com/science/article/pii/S0146641014000878

2. BaBar, J.P. Lees et al., Time-Integrated Luminosity Recorded by the BABAR Detector at the PEP-II $e^{+} e^{-}$Collider, Nucl. Instrum. Meth. A 726, 203-213 (2013). arXiv:1301.2703 [hep-ex]

3. Belle-II, F. Abudinén et al., Measurement of the integrated luminosity of the Phase 2 data of the Belle II experiment, Chin. Phys. C 44 no. 2, 021001 (2020). arXiv:1910.05365 [hep-ex]

4. H.J. Bhabha, The scattering of positrons by electrons with exchange on Dirac's theory of the positron. Proc. Roy. Soc. Lond. A 154, 195-206 (1936)

5. I.C. Brock et al., Luminosity measurement in the L3 detector at LEP. Nucl. Instrum. Meth. A 381, 236-266 (1996)

6. T. Camporesi, V. Obratzsov, M. Paganoni, F. Terranova, M. Bigi, I. Gouz, E. Migliore, B. Tome, Luminosity measurement in 1994 with the STIC detector. DELPHI-97-8 PHYS 667, (1997). https://cds.cern. ch/record/2629467

7. OPAL Collaboration, G. Abbiendi et al., Precision luminosity for $\mathrm{Z}^{0}$ lineshape measurements with a silicon-tungsten calorimeter. Eur. Phys. J. C 14, 373-425 (2000)

8. D. Bédérède, et al., SICAL - a high precision silicon-tungsten luminosity calorimeter for ALEPH, Nucl. Inst. Meth. A365 no. 1, 117-134 (1995). http://www.sciencedirect.com/science/article/pii/ 0168900295004092

9. A. Blondel, P. Janot, FCC-ee overview: new opportunities create new challenges, in A future Higgs and Electroweak factory (FCC): Challenges towards discovery, EPJ+ special issue, Focus on FCC-ee, arXiv:2106.13885 [hep-ex]

10. J. Alcaraz Maestre, A. Blondel, M. Dam, P. Janot, The Z lineshape challenge: ppm and keV measurements, Eur. Phys. J. Plus 136, 848 (2021). arXiv:2107.00616 [hep-ex], https://doi.org/10.1140/epjp/s13360-02101760-X

11. P. Azzurri, The W mass and width measurement challenge at FCC-ee, Eur. Phys. J. Plus 136, 1203 (2021). arXiv:2107.04444 [hep-ex], https://doi.org/10.1140/epjp/s13360-021-02211-3

12. S. Jadach, W. Płaczek, M. Skrzypek, B. Ward, S. Yost, The path to $0.01 \%$ theoretical luminosity precision for the FCC-ee. Phys. Lett. B. 790, 314-321 (2019). https://www.sciencedirect.com/science/article/pii/ S0370269319300280

13. A. Abada et al., FCC-ee: The Lepton Collider, Eur. Phys. J. Special Topics 228 no. 2, 261-623 (2019). https://doi.org/10.1140/epjst/e2019-900045-4

14. M. Boscolo et al., Challenges for the interaction region design of the Future Circular Collider FCC-ee, in 12th International Particle Accelerator Conference. (2021), arXiv:2105.09698 [physics.acc-ph]

15. S. Jadach, W. Placzek, E. Richter-Waas, B. Ward, Z. Was, Upgrade of the Monte Carlo program BHLUMI for Bhabha scattering at low angles to version 4.04, Comput. Phys. Commun.102 no. 1, 229-251 (1997). http://www.sciencedirect.com/science/article/pii/S0010465596001567

16. H. Abramowicz et al., The International Linear Collider Technical Design Report - Volume 4: Detectors, arXiv:1306.6329 [physics.ins-det]

17. M. Aicheler et al., A Multi-TeV Linear Collider Based on CLIC Technology: CLIC Conceptual Design Report, Tech. Rep. CERN-2012-007. SLAC-R-985. KEK-Report-2012-1. PSI-12-01. JAI-2012-001, Geneva, 2012. https://cds.cern.ch/record/1500095

18. D. Schulte, Beam-Beam Simulations with GUINEA-PIG, https://cds.cern.ch/record/382453

19. E. Currás et al., Radiation hardness and precision timing study of silicon detectors for the CMS high granularity calorimeter (HGC). Nucl. Instrum. Meth. A 845, 60-63 (2017)

20. J. Crawford, E. Hughes, L. O'Neill, R. Rand, A precision luminosity monitor for use at electron-positron storage rings, Nuclear Instruments and Methods 127 no. 2, 173-182 (1975). http://www.sciencedirect. com/science/article/pii/0029554X75904851

21. G. Voutsinas, E. Perez, M. Dam, P. Janot, Beam-beam effects on the luminosity measurement at FCC-ee. JHEP 10, 225 (2019). arXiv:1908.01698 [hep-ex]

22. G. Voutsinas, E. Perez, M. Dam, P. Janot, Beam-beam effects on the luminosity measurement at LEP and the number of light neutrino species. Phys. Lett. B 800, 135068 (2020). arXiv:1908.01704 [hep-ex] 